ANNALES

POLONICI MATHEMATICI

$86.2(2005)$

\title{
Existence of solutions for infinite systems of parabolic equations with functional dependence
}

\author{
by Anna PudeŁko (Kraków)
}

\begin{abstract}
The Cauchy problem for an infinite system of parabolic type equations is studied. General operators of parabolic type of second order with variable coefficients are considered and the system is weakly coupled. We prove the existence and uniqueness of a bounded solution under Carathéodory type conditions and its differentiability, as well as the existence and uniqueness in the class of functions satisfying a natural growth condition. Both results are obtained by the fixed point method.
\end{abstract}

1. Introduction. Let $S$ be an arbitrary set of indices. Let $B(S)$ be the space of mappings $v: S \ni i \mapsto v^{i} \in \mathbb{R}$ such that $\sup \left\{\left|v^{i}\right|: i \in S\right\}<\infty$ endowed with the supremum norm

$$
\|v\|_{B(S)}:=\sup \left\{\left|v^{i}\right|: i \in S\right\} .
$$

For every nonempty set $X \subset \mathbb{R}^{m}$ we denote by $C_{S}(X)$ the space of mappings $w: X \ni x \mapsto w(x) \in B(S)$, where $w(x): S \ni i \mapsto w^{i}(x) \in \mathbb{R}$, and the functions $w^{i}$ are continuous in $X$. We also use the notation $w=\left\{w^{i}\right\}_{i \in S}$. When the functions $w^{i}$ are additionally bounded we denote the space of these functions by $C B_{S}(X)$. We remark that the space $C B_{S}(X)$ endowed with the supremum norm

$$
\|w\|_{0}:=\sup \left\{\left|w^{i}(x)\right|: x \in X, i \in S\right\}
$$

is a Banach space.

Let $T$ be an arbitrary positive constant. Set $\Omega=(0, T] \times \mathbb{R}^{m}, \bar{\Omega}=$ $[0, T] \times \mathbb{R}^{m}$. For $w \in C B_{S}(\bar{\Omega})$ and for a fixed $t \geq 0$ we define

$$
\|w\|_{0, t}:=\sup \left\{\left|w^{i}(\widetilde{t}, x)\right|:(\widetilde{t}, x) \in \bar{\Omega}, \widetilde{t} \leq t, i \in S\right\} .
$$

2000 Mathematics Subject Classification: Primary 35K15; Secondary 35K55, 35R10, $47 \mathrm{H} 10$.

Key words and phrases: infinite systems, parabolic differential-functional equations, Cauchy problem, Banach fixed point theorem.

Part of this work is supported by local Grant No. 11.420.04. 
Let $f=\left\{f^{i}\right\}_{i \in S}$ and $\varphi=\left\{\varphi^{i}\right\}_{i \in S}$, where

$$
\begin{gathered}
f^{i}: \bar{\Omega} \times C_{S}(\bar{\Omega}) \ni(t, x, s) \mapsto f^{i}(t, x, s) \in \mathbb{R}, \quad i \in S, \\
\varphi^{i}: \mathbb{R}^{m} \ni x \mapsto \varphi^{i}(x) \in \mathbb{R}, \quad i \in S .
\end{gathered}
$$

Let $u=\left\{u^{i}\right\}_{i \in S}$ be composed of unknown functions $u^{i}$ of the variables $(t, x)=\left(t, x_{1}, \ldots, x_{m}\right) . \mathcal{F}=\left\{\mathcal{F}^{i}\right\}_{i \in S}$ denotes the family of the following second order parabolic operators:

$$
\mathcal{F}^{i}:=\frac{\partial}{\partial t}-\mathcal{A}^{i}, \quad \mathcal{A}^{i}:=\sum_{j, k=1}^{m} a_{j k}^{i}(t, x) \frac{\partial^{2}}{\partial x_{j} \partial x_{k}}+\sum_{j=1}^{m} b_{j}^{i}(t, x) \frac{\partial}{\partial x_{j}}+c^{i}(t, x),
$$

where the coefficients $a_{j k}^{i}(t, x), b_{j}^{i}(t, x)$ and $c^{i}(t, x)$ are defined in $\bar{\Omega}$.

We consider an infinite system of weakly coupled $\left({ }^{1}\right)$ semilinear parabolic equations of reaction-diffusion-convection type of the form

$$
\mathcal{F}^{i}\left[u^{i}\right](t, x)=f^{i}(t, x, u), \quad i \in S,
$$

supplemented with the initial condition

$$
u(0, x)=\varphi(x) \quad \text { for } x \in \mathbb{R}^{m} .
$$

The notation $f(t, x, u)$ means that the functions $f^{i}$ are functionals of the function $u$. We consider the functional dependence of Volterra type, i.e.

$(\mathcal{V})$ for any $(t, x) \in \Omega$ and $\eta, \widetilde{\eta} \in C_{S}(\bar{\Omega})$ such that $\eta^{j}(\bar{t}, x)=\widetilde{\eta}^{j}(\bar{t}, x)$ for $0 \leq \bar{t} \leq t, j \in S$ we have $f^{i}(t, x, \eta)=f^{i}(t, x, \widetilde{\eta}), i \in S$.

This means that the values of the functions $f^{i}(t, x, u), i \in S$, depend only on the past history of the process. Examples of such functionals are:

$$
f^{i}(t, x, u)=u^{i+1}\left(\alpha^{i} t, \beta^{i} x\right)
$$

where $\alpha^{i} \in[0,1], \beta^{i} \in \mathbb{R}^{m}$ and $\beta^{i} x=\left(\beta_{1}^{i} x_{1}, \ldots, \beta_{m}^{i} x_{m}\right)$;

$$
f^{i}(t, x, u)= \begin{cases}u^{i+1}(t-\tau, x) & \text { for } 0 \leq \tau \leq t, x \in \mathbb{R}^{m}, \\ \alpha(t-\tau, x) & \text { for } 0 \leq t<\tau, x \in \mathbb{R}^{m}\end{cases}
$$

where $\alpha=\left\{\alpha^{i}\right\}_{i \in S}$ is a function defined for $[-\tau, 0] \times \mathbb{R}^{m}$;

$$
f^{i}(t, x, u)=\int_{0}^{t} A^{i}(\tau, x, u(\tau, x)) d \tau
$$

Such functionals can describe delays and deviations or be integrals "over the past".

This paper can be considered as a continuation of the author's study [10] of certain infinite systems of parabolic differential-functional equations. Now, we consider a more general form of operator with lower order $x$-derivatives.

$\left({ }^{1}\right)$ That is, every equation contains all unknown functions and derivatives of only one unknown function. 
The goal of the paper is to prove two theorems. The first concerns the existence and uniqueness of solution of the Cauchy problem (1), (2) for the infinite system in the class of bounded continuous functions under Carathéodory type assumptions, i.e. the integrability of the function $f$ with respect to $t$ and continuity with respect to the other variables. The second is a similar theorem but in the class of functions satisfying a certain growth condition. We will apply the Banach contraction principle, as in [10], [3], [4]. In [10], to solve the above problem in the space of bounded continuous functions under the classical assumptions the Banach fixed point theorem was also used. In [3] and [4], by the same methods, the existence and uniqueness of solution was proved for the Cauchy problem for a parabolic equation with functional dependence represented by a Hale-type operator. The initial-boundary value problem for similar infinite systems was considered e.g. in [1], [2].

This paper is organized as follows. In the next section the necessary notations and definitions are introduced. We also formulate the assumptions which are valid throughout the paper, and recall two auxiliary lemmas. Section 3 contains the results concerning the bounded continuous solutions. In the last section we state and prove the main result of the paper, i.e. the existence and uniqueness of solution for the Cauchy problem (1), (2) with the growth condition $\left|u^{i}(t, x)\right| \leq D \exp \left(d|x|^{2}\right)$.

2. Notations, definitions and assumptions. Throughout the paper we use the following notation. The Euclidean norm in $\mathbb{R}^{m}$ is denoted by $|\cdot|$ and the norms in function spaces by ||$\cdot||$ with appropriate indices; in particular the supremum norm is denoted by $\|\cdot\|_{0}$. By $L^{1}(X)$ we denote the space of all integrable functions defined on a nonempty measurable set $X \subset \mathbb{R}^{m}$.

We now formulate the crucial assumptions on the coefficients of the operators $\left\{\mathcal{F}^{i}\right\}_{i \in S}$, which are sufficient for existence of a fundamental solution for the homogeneous system associated with (1).

$(\mathcal{H}) \quad$ The coefficients $a_{j k}^{i}(t, x), b_{j}^{i}(t, x), c^{i}(t, x), i \in S, j, k=1, \ldots, m$, are bounded continuous functions in $\bar{\Omega}$ such that $a_{j k}^{i}(t, x)=a_{k j}^{i}(t, x)$ and the following uniform Hölder conditions with exponent $\alpha(0<\alpha \leq 1)$ are satisfied in $\bar{\Omega}$ : there exists $H>0$ such that

$$
\begin{aligned}
\left|a_{j k}^{i}(t, x)-a_{j k}^{i}\left(t^{\prime}, x^{\prime}\right)\right| & \leq H\left(\left|x-x^{\prime}\right|^{\alpha}+\left|t-t^{\prime}\right|^{\alpha / 2}\right), \\
\left|b_{j}^{i}(t, x)-b_{j}^{i}\left(t, x^{\prime}\right)\right| & \leq H\left|x-x^{\prime}\right|^{\alpha}, \\
\left|c^{i}(t, x)-c^{i}\left(t, x^{\prime}\right)\right| & \leq H\left|x-x^{\prime}\right|^{\alpha},
\end{aligned}
$$

for all $(t, x),\left(t^{\prime}, x^{\prime}\right) \in \bar{\Omega}$ and $j, k=1, \ldots, m$.

( $\mathcal{P})$ The operators $\mathcal{F}^{i}, i \in S$, are uniformly parabolic in $\bar{\Omega}$, i.e. there is $\mu>0$ such that 


$$
\sum_{j, k=1}^{m} a_{j k}^{i}(t, x) \xi_{j} \xi_{k} \geq \mu \sum_{j=1}^{m} \xi_{j}^{2}
$$

for all $(t, x) \in \bar{\Omega}$ and $\xi=\left(\xi_{1}, \ldots, \xi_{m}\right) \in \mathbb{R}^{m}$.

LEMMA 1. If assumptions $(\mathcal{P})$ and $(\mathcal{H})$ hold then there exist fundamental solutions $\Gamma^{i}(t, x ; \tau, \xi)$ of the equations $\mathcal{F}^{i}\left[u^{i}\right](t, x)=0, i \in S$, and the following inequalities hold:

$$
\left|\Gamma^{i}(t, x ; \tau, \xi)\right| \leq c(t-\tau)^{-m / 2} \exp \left(-\frac{\mu^{*}|x-\xi|^{2}}{4(t-\tau)}\right), \quad i \in S,
$$

for some $\mu^{*}<\mu$ where $\mu^{*}$ depends on $\mu$ and $H$ whereas $c$ depends on $\mu, \alpha, T$.

A proof of Lemma 1 can be found in [6, Chap. 1, Th. 2.1] or [7, Chap. 1, Sec. 2-6].

REMARK 1. Under the assumptions of Lemma 1 the following estimates on the derivatives of the fundamental solution hold:

$$
\begin{gathered}
\left|\frac{\partial}{\partial x_{j}} \Gamma^{i}(t, x ; \tau, \xi)\right| \leq \frac{c_{1}}{(t-\tau)^{(m+1) / 2}} \exp \left(-\mu^{*} \frac{|x-\xi|^{2}}{4(t-\tau)}\right), \\
\left|\frac{\partial}{\partial t} \Gamma^{i}(t, x ; \tau, \xi)\right|,\left|\frac{\partial^{2}}{\partial x_{j} \partial x_{k}} \Gamma^{i}(t, x ; \tau, \xi)\right| \leq \frac{c_{2}}{(t-\tau)^{(m+2) / 2}} \exp \left(-\mu^{*} \frac{|x-\xi|^{2}}{4(t-\tau)}\right)
\end{gathered}
$$

for all $0 \leq \tau<t \leq T, x, \xi \in \mathbb{R}^{m}, i \in S$ and $j, k=1, \ldots, m$.

We can also obtain Hölder continuity conditions with $\delta \in(0,1]$ :

$$
\begin{aligned}
& \mid \Gamma^{i}(t, x ; \tau, \xi)-\Gamma^{i}(\bar{t}, \bar{x} ; \tau, \xi) \mid \\
& \leq \frac{c_{0, \delta}}{(t-\tau)^{(m+\delta) / 2}} \exp \left(-\mu^{*} \frac{|x-\xi|^{2}}{4(t-\tau)}\right)\left[|t-\bar{t}|^{\delta / 2}+|x-\bar{x}|^{\delta}\right] \\
&\left|\frac{\partial}{\partial x_{j}} \Gamma^{i}(t, x ; \tau, \xi)-\frac{\partial}{\partial x_{j}} \Gamma^{i}(\bar{t}, \bar{x} ; \tau, \xi)\right| \\
& \leq \frac{c_{1, \delta}}{(t-\tau)^{(m+1+\delta) / 2}} \exp \left(-\mu^{*} \frac{|x-\xi|^{2}}{4(t-\tau)}\right)\left[|t-\bar{t}|^{\delta / 2}+|x-\bar{x}|^{\delta}\right]
\end{aligned}
$$

for all $0 \leq \tau<\bar{t} \leq t \leq T, x, \bar{x}, \xi \in \mathbb{R}^{m}, i \in S$ and $j, k=1, \ldots, m$. A proof of this can be found in [6, Chap. 1].

The above inequalities imply immediately

$$
\int_{\mathbb{R}^{m}}\left|\Gamma^{i}(t, x ; \tau, \xi)\right| d \xi \leq \text { and } \int_{\mathbb{R}^{m}}\left|\frac{\partial}{\partial x_{j}} \Gamma^{i}(t, x ; \tau, \xi)\right| d \xi \leq C_{1}(t-\tau)^{-1 / 2}
$$

for $i \in S, j=1, \ldots, m$, where $C=c\left(4 \pi / \mu^{*}\right)^{m / 2}$ and $C_{1}=c_{1}\left(4 \pi / \mu^{*}\right)^{m / 2}$. The notation for constants which appear in Lemma 1 and Remark 1 will be valid throughout the paper. 
Now, for each $\eta \in C_{S}(\bar{\Omega})$ we define the operator $\mathbf{F}=\left\{\mathbf{F}^{i}\right\}_{i \in S}$ by setting $\mathbf{F}^{i}[\eta](t, x):=f^{i}(t, x, \eta), \quad i \in S$.

Using the fundamental solutions and the operator $\mathbf{F}$ we can transform the differential problem (1), (2) into the integral system

$$
\begin{array}{r}
u^{i}(t, x)=\int_{\mathbb{R}^{m}} \Gamma^{i}(t, x ; 0, \xi) \varphi^{i}(\xi) d \xi+\int_{0}^{t} \int_{\mathbb{R}^{m}} \Gamma^{i}(t, x ; \tau, \xi) \mathbf{F}^{i}[u](\tau, \xi) d \xi d \tau \\
\text { for } t>0, x \in \mathbb{R}^{m} .
\end{array}
$$

Definition. A function $u \in C_{S}(\bar{\Omega})$ is said to be a $C$-solution of the differential problem (1), (2) in $\bar{\Omega}$ if it satisfies the system of integral equations (3).

To end this section we recall a certain technical lemma which will be used later.

Lemma 2. If $0 \leq B<A$ then

$$
\int_{\mathbb{R}^{m}} \exp \left(-A|x-\xi|^{2}+B|\xi|^{2}\right) d \xi=\left(\frac{\pi}{A-B}\right)^{m / 2} \exp \left(\frac{A B}{A-B}|x|^{2}\right) .
$$

This follows from the equality

$$
-A|x-\xi|^{2}+B|\xi|^{2}=\sum_{i=1}^{n} \frac{A B}{A-B} x_{i}^{2}-\left(\sqrt{A-B} \xi_{i}-\frac{A}{\sqrt{A-B}} x_{i}^{2}\right)^{2} .
$$

Changing variables as follows: $z_{i}=\sqrt{A-B} \xi_{i}-\frac{A}{\sqrt{A-B}} x_{i}^{2}$, and making use of the fact that $\int_{\mathbb{R}^{m}} \exp \left(-|z|^{2}\right) d z=\pi^{m / 2}$ for $z=\left(z_{1}, \ldots, z_{m}\right)$, we obtain the result.

3. Bounded solutions. Recall that in [10] we proved the existence and uniqueness of a bounded solution for the problem (1), (2). The main idea of the proof was as follows. In the space $C B_{S}(\bar{\Omega})$ we defined an operator $\mathbf{T}$ by the right-hand sides of the integral system (3). Then using the Banach fixed point theorem we found the unique solution of the equation $u=\mathbf{T}[u]$ in that space with respect to a weighted norm $\|\cdot\|_{0, \psi}$ (of Bielecki type, cf. [5]). The result was obtained under the classical assumptions, i.e.

Assumption 1.

(C) $f^{i}(\cdot, \cdot, s)$ is continuous for all $i$ and $s$;

(L) $f^{i}$ satisfies the Lipschitz condition in $s$ uniformly with respect to $(t, x)$ and i, i.e. $\exists L>0 \forall i \in S \forall(t, x) \in \bar{\Omega} \forall s, \widetilde{s} \in C B_{S}(\bar{\Omega})$

$$
\left|f^{i}(t, x, s)-f^{i}(t, x, \widetilde{s})\right| \leq L\|s-\widetilde{s}\|_{0}
$$

(B) $\exists M_{0}>0 \forall i \in S \forall(t, x) \in \bar{\Omega}\left|f^{i}(t, x, 0)\right| \leq M_{0}$. 
The definition of $C$-solution and analysis of the classical results suggest that it is possible to obtain similar existence theorems under weaker conditions. Namely, one can replace continuity conditions by Carathéodory type conditions, i.e. integrability of the right-hand sides with respect to $t$, and replace the Lipschitz constants in the classical Lipschitz condition by suitable integrable functions. This approach admits a weak singularity at $t=0^{+}$, that is, we can consider $L(t)=t^{-\beta}, \beta \in[0,1)$.

Now, we formulate the Carathéodory type conditions.

Assumption 2.

$\left(\mathbf{C}_{t}\right) f^{i}(t, \cdot, s) \in C\left(\mathbb{R}^{m}\right)$ and $f^{i}(\cdot, x, s) \in L^{1}[0, T]$ for all $i$;

$\left(\mathbf{L}_{t}\right) f^{i}$ satisfies the Lipschitz condition in $s$ uniformly with respect to $(t, x)$ and i, i.e. $\exists L \in L^{1}[0, T] \forall i \in S \forall(t, x) \in \bar{\Omega} \forall s, \widetilde{s} \in C B_{S}(\bar{\Omega})$

$$
\left|f^{i}(t, x, s)-f^{i}(t, x, \widetilde{s})\right| \leq L(t)\|s-\widetilde{s}\|_{0} ;
$$

$\left(\mathbf{B}_{t}\right) \exists M_{0} \in L^{1}[0, T] \forall i \in S \forall(t, x) \in \bar{\Omega}\left|f^{i}(t, x, 0)\right| \leq M_{0}(t)$.

An example of $f=\left\{f^{i}\right\}_{i \in S}$ which satisfies these conditions is

$$
f^{i}(t, x, u)=\int_{0}^{t / 2} m(t-\tau) A^{i}(u(t, x)) d \tau
$$

where $A=\left\{A^{i}\right\}_{i \in S}$ satisfies the Lipschitz condition with respect to the norm $\|\cdot\|_{B(S)}$ uniformly with respect to $i \in S$ and $\sup _{i \in S}\left|A^{i}(0)\right|<\infty$, and $m$ is a function such that $t \mapsto \int_{0}^{t / 2}|m(t-\tau)| d \tau$ is integrable on [0,T]; e.g. $m(t)=1 / t^{1+\beta}$ with $\beta \in(0,1)$ and $A^{i}=\sum_{j=i-k}^{i+k} a_{j} z^{i}$ where $k$ is a positive integer and $\left\{a_{n}\right\}_{n \in \mathbb{N}}$ is a bounded sequence.

Theorem 1. Let assumptions $(\mathcal{H})$ and $(\mathcal{P})$ hold. Let $f=\left\{f^{i}\right\}_{i \in S}$ satisfy Assumption 2 and $\varphi \in C B_{S}\left(\mathbb{R}^{m}\right)$. Then there exists a unique $C$-solution $u$ of the problem (1), (2) in $\bar{\Omega}$.

Proof. The proof is analogous to that in [10]. Let $\psi(t)$ be a nondecreasing continuous function defined on $[0, T]$ which satisfies the inequality

$$
\int_{0}^{t} C L(\tau) \psi(\tau) d \tau \leq \theta \psi(t), \quad 0<\theta<1
$$

for example $\psi(t)=\exp \left\{(C / \theta) \int_{0}^{t} L(\tau) d \tau\right\}$. Let the operator $\mathbf{T}=\left\{\mathbf{T}^{i}\right\}_{i \in S}$ be defined by the right-hand side of (3), i.e. for $t>0, x \in \mathbb{R}^{m}$,

$$
\mathbf{T}^{i}[u](t, x)=\int_{\mathbb{R}^{m}} \Gamma^{i}(t, x ; 0, \xi) \varphi^{i}(\xi) d \xi+\int_{0}^{t} \int_{\mathbb{R}^{m}} \Gamma^{i}(t, x ; \tau, \xi) \mathbf{F}^{i}[u](\tau, \xi) d \xi d \tau .
$$


For $u=\mathbf{T}[z], \bar{u}=\mathbf{T}[\bar{z}]$, and $z, \bar{z} \in C B_{S}(\bar{\Omega})$ we have

$$
\left|u^{i}(t, x)-\bar{u}^{i}(t, x)\right| \leq\|z-\bar{z}\|_{0, \psi} \int_{0}^{t} C L(\tau) \psi(\tau) d \tau \leq \theta\|z-\bar{z}\|_{0, \psi} \psi(t) .
$$

This shows that $\mathbf{T}$ defines a contraction from the space $C B_{S}(\bar{\Omega})$ into itself with respect to the weighted norm $\|\cdot\|_{0, \psi}$, where

$$
\|z\|_{0, \psi}:=\sup \left\{\frac{\left|z^{i}(t, x)\right|}{\psi(t)}:(t, x) \in \bar{\Omega}, i \in S\right\} .
$$

The boundedness of $\mathbf{T}[z]$ is an easy consequence of Assumption 2 and the estimate

$$
\|\mathbf{T}[z]\|_{0} \leq C\left(\bar{K}+\|z\|_{0} \int_{0}^{t} L(\tau) \psi(\tau) d \tau+\int_{0}^{t} M_{0}(\tau) \psi(\tau) d \tau\right) .
$$

Next, we recall that the $C$-solution of the problem (1), (2) in $\bar{\Omega}$ obtained under classical assumptions has continuous first order $x$-derivatives in $\Omega$ with no additional assumption.

In case of Carathéodory type assumptions, it is also possible to obtain continuous $x$-differentiability, but under stronger assumptions.

First, we introduce the following notation:

$$
u_{\varphi}(t, x):=\int_{\mathbb{R}^{m}} \Gamma^{i}(t, x ; 0, \xi) \varphi^{i}(\xi) d \xi .
$$

Proposition 1. Suppose that all assumptions of Theorem 1 hold and $\left(\mathbf{B}_{\varphi}\right) \exists M_{\varphi} \in L^{1}[0, T] \forall i \in S \forall(t, x) \in \bar{\Omega}\left|f^{i}\left(t, x, u_{\varphi}\right)\right| \leq M_{\varphi}(t)$.

Then the $C$-solution u satisfies the inequality

$$
\left\|u-u_{\varphi}\right\|_{0, t} \leq G(t):=\int_{0}^{t} C M_{\varphi}(\tau) \exp \left(C \int_{\tau}^{t} L(s) d s\right) d \tau
$$

Furthermore, if

$$
\exists M>0 \exists \delta \in(0,1) \forall t \in[0, T] \quad \int_{0}^{t} \frac{M_{\varphi}(\tau)+L(\tau) G(\tau)}{(t-\tau)^{(\delta+1) / 2}} d \tau<M
$$

and

$$
\lim _{h \searrow 0} h \int_{0}^{t-h^{2}}\left(M_{\varphi}(\tau)+L(\tau) G(\tau)\right)(t-\tau)^{-1} d \tau=0, \quad t>0,
$$

then the first order $x$-derivatives of $u$ in $\Omega$ exist and are continuous. 
Proof. To obtain (4) we estimate the difference $u-u_{\varphi}$ in the same way as we proved the boundedness of $\mathbf{T}[z]$ in the previous theorem:

$$
\begin{aligned}
& \left|\left(u-u_{\varphi}\right)(t, x)\right| \leq \int_{0 \mathbb{R}^{m}}^{t}\left|\Gamma^{i}(t, x ; \tau, \xi) f^{i}(\tau, \xi, u)\right| d \xi d \tau \\
& \quad \leq \int_{0 \mathbb{R}^{m}}^{t}\left|\Gamma^{i}(t, x ; \tau, \xi)\right|\left(\left|\mathbf{F}^{i}[u](\tau, \xi)-\mathbf{F}^{i}\left[u_{\varphi}\right](\tau, \xi)\right|+\left|\mathbf{F}^{i}\left[u_{\varphi}\right](\tau, \xi)\right|\right) d \xi d \tau .
\end{aligned}
$$

Then by $\left(\mathbf{B}_{\varphi}\right),\left(\mathbf{L}_{t}\right)$, and $(\mathcal{V})$,

$$
\left|\left(u-u_{\varphi}\right)(t, x)\right| \leq \int_{0}^{t} C\left(M_{\varphi}(\tau)+L(\tau)\left\|u-u_{\varphi}\right\|_{0, \tau}\right) d \tau,
$$

and the Gronwall lemma yields (4).

Define

$$
I_{j}^{i}:=\int_{\mathbb{R}^{m}} \frac{\partial}{\partial x_{j}} \Gamma^{i}(t, x ; \tau, \xi) \mathbf{F}^{i}[u](\tau, \xi) d \xi \quad \text { for } i \in S, j=1, \ldots, m .
$$

By Remark 1 , for all $i \in S$ and $j=1, \ldots, m$,

$$
\begin{aligned}
\left|I_{j}^{i}\right| & \leq \int_{\mathbb{R}^{m}} \frac{c_{1}}{(t-\tau)^{m / 2}} \exp \left\{-\mu_{*} \frac{(x-\xi)^{2}}{t-\tau}\right\} \frac{1}{\sqrt{t-\tau}}\left(M_{\varphi}(\tau)+L(\tau) G(\tau)\right) d \xi \\
& \leq \frac{C_{1}}{\sqrt{t-\tau}}\left(M_{\varphi}(\tau)+L(\tau) G(\tau)\right) .
\end{aligned}
$$

From (5) it follows that the integrals $\int_{0}^{t} I_{j}^{i} d \tau$ are almost uniformly convergent for all $t, x$ where $(t, x) \in \Omega$, which implies the differentiability of $u$ with respect to $x$. Their continuity can be obtained from the estimate

$$
\begin{aligned}
\left|\int_{0}^{t} \int_{\mathbb{R}^{m}} \frac{\partial}{\partial x_{j}} \Gamma^{i}(t, x ; \tau, \xi) \mathbf{F}^{i}[u](\tau, \xi) d \xi d \tau-\int_{0 \mathbb{R}^{m}}^{\bar{t}} \frac{\partial}{\partial x_{j}} \Gamma^{i}(\bar{t}, \bar{x} ; \tau, \xi) \mathbf{F}^{i}[u](\tau, \xi) d \xi d \tau\right| \\
\leq c_{1,1}\left(\frac{4 \pi}{\mu^{*}}\right)^{m / 2} \int_{0}^{\bar{t}}\left(M_{\varphi}(\tau)+L(\tau) G(\tau)\right) \frac{\sqrt{t-\bar{t}}}{t-\tau} d \tau \\
+C_{1} \int_{\bar{t}}^{t}\left(M_{\varphi}(\tau)+L(\tau) G(\tau)\right) \frac{1}{\sqrt{t-\tau}} d \tau \\
+c_{1, \delta}\left(\frac{4 \pi}{\mu^{*}}\right)^{m / 2} \int_{0}^{\bar{t}}\left(M_{\varphi}(\tau)+L(\tau) G(\tau)\right) \frac{|x-\bar{x}|^{\delta}}{(t-\tau)^{(1+\delta) / 2}} d \tau
\end{aligned}
$$

for all $0 \leq \bar{t}<t \leq T, x, \bar{x}, \xi \in \mathbb{R}^{m}, i \in S$ and $j, k=1, \ldots, m$. 
Thus, taking $h=\sqrt{t-\bar{t}}$ and making use of (5) and (6) one can see that $\int_{0}^{t} I_{j}^{i} d \tau$ are continuous in $x$ and continuous in $t$ uniformly with respect to $x$ for all $i \in S$ and $j=1, \ldots, m$. This implies the continuity of the first order $x$-derivatives of the $C$-solution and ends the proof of Proposition 1.

4. Unbounded solutions. This section is devoted to the existence of a unique solution for the problem (1), (2) in the class of continuous functions which satisfy the growth condition $\left|u^{i}(t, x)\right| \leq D \exp \left(d|x|^{2}\right)$. Without this condition, the initial-valued problem in an unbounded domain is ill-posed, as shown by Tikhonov's well known example [11]. The results in this section are similar to those concerning bounded solutions, but there is an important difference: the solutions in the previous section were global in time, whereas if the initial data and right-hand sides are unbounded then the solutions may blow up.

We denote by $C^{+}$the space of all positive, real-valued, continuous and nondecreasing functions defined on the set $[0, T]$.

For $w \in C_{S}(\bar{\Omega})$ we define the following family of weighted norms of Bielecki type:

$$
\begin{aligned}
\|w\|_{2, \phi} & :=\sup _{i \in S} \sup _{(t, x) \in \bar{\Omega}} \frac{\left|w^{i}(t, x)\right|}{\exp \left(\phi(t)|x|^{2}\right)} \\
\|w\|_{2, \phi, \psi} & :=\sup _{i \in S} \sup _{(t, x) \in \bar{\Omega}} \frac{\left|w^{i}(t, x)\right|}{\psi(t)[\phi(t)]^{m / 2} \exp \left(\phi(t)|x|^{2}\right)},
\end{aligned}
$$

where $\phi, \psi \in C^{+}$. Let $E_{S}^{2, \phi}$ be the space of all functions $w \in C_{S}(\bar{\Omega})$ such that

$$
\exists D \geq 0 \forall(t, x) \in \bar{\Omega} \forall i \in S \quad\left|w^{i}(t, x)\right| \leq D \exp \left(\phi(t)|x|^{2}\right)
$$

for every $\phi \in C^{+}$. We notice that the space $E_{S}^{2, \phi}$ endowed with the norm $\|\cdot\|_{2, \phi}$ is a Banach space. If $\psi, \phi \in C^{+}$, then

$$
\psi(0)[\phi(0)]^{n / 2}\|w\|_{2, \phi, \psi} \leq\|w\|_{2, \phi} \leq \psi(T)[\phi(T)]^{n / 2}\|w\|_{2, \phi, \psi},
$$

which shows that the weighted norms $\|\cdot\|_{2, \phi}$ and $\|\cdot\|_{2, \phi, \psi}$ are equivalent. This immediately implies that $E_{S}^{2, \phi}$ with the norm $\|\cdot\|_{2, \phi, \psi}$ is a Banach space as well.

Assumption 3.

$(\mathcal{K}) \exists \bar{K} \geq 0 \forall i \in S \forall x \in \mathbb{R}^{m}\left|\varphi^{i}(x)\right| \leq \bar{K} \exp \left(\phi(0)|x|^{2}\right)$;

(C) $f^{i}(\cdot, \cdot, s)$ is continuous for each $i \in S$;

(B) $\exists M_{0} \geq 0 \forall i \in S \forall(t, x) \in \bar{\Omega}\left|f^{i}(t, x, 0)\right| \leq M_{0} \exp \left(\phi(t)|x|^{2}\right)$.

An example of $f=\left\{f^{i}\right\}_{i \in S}$ which satisfies the conditions from Assumption 3 and condition $(\mathcal{L})$ which appears in the assumptions of Theorem 2 is 


$$
f^{i}(t, x, u)=\int_{0}^{t} m(t-\tau) A^{i}(u(t, x)) d \tau
$$

where $m$ is a function such that $\int_{0}^{T}|m(T-\tau)| d \tau<\infty$ and $A=\left\{A^{i}\right\}_{i \in S}$ satisfies the Lipschitz condition with respect to the norm $\|\cdot\|_{B(S)}$ uniformly with respect to $i \in S$ and $\sup _{i \in S}\left|A^{i}(0)\right|<\infty$.

Theorem 2. Let assumptions $(\mathcal{H})$ and $(\mathcal{P})$ hold. Let $\phi \in C^{+}$be a function satisfying the inequality

$$
\frac{\mu^{*} \phi(\tau)}{\mu^{*}-4 \phi(\tau)(t-\tau)} \leq \phi(t) \quad \text { for } 0 \leq \tau \leq t \leq T,
$$

where $\mu^{*}$ is the constant which appeared in Lemma 1. Let Assumption 3 be satisfied and let $\psi_{L}(t):=\exp \{C L t / \theta\}$, where $0<\theta<1, L>0$. Moreover, suppose that the operator generated by $f=\left\{f^{i}\right\}_{i \in S}$ satisfies the following condition:

$$
(\mathcal{L}) \exists L>0 \forall i \in S \forall s, \widetilde{s} \in E_{S}^{2, \phi}
$$

$$
\left\|\mathbf{F}^{i}[s]-\mathbf{F}^{i}[\widetilde{s}]\right\|_{2, \phi, \psi_{L}} \leq L\|s-\widetilde{s}\|_{2, \phi, \psi_{L}} .
$$

Then there exists a unique solution $u \in E_{S}^{2, \phi}$ of the problem (1), (2).

REMARK 2. In order to avoid the dependence of the left hand side on $L$, condition $(\mathcal{L})$ can be written in the following equivalent form:

$$
\begin{aligned}
& \left(\mathcal{L}^{\prime}\right) \exists L>0 \forall i \in S \forall s, \widetilde{s} \in E_{S}^{2, \phi} \\
& \quad\left|f^{i}(t, x, s)-f^{i}(t, x, \widetilde{s})\right| \leq L\|s-\widetilde{s}\|_{2, \phi, \psi_{L}} \psi_{L}(t)[\phi(t)]^{m / 2} \exp \left(\phi(t)|x|^{2}\right) .
\end{aligned}
$$

Before giving a proof of Theorem 2 we give an example of a function $\phi(t)$ satisfying the assumptions (cf. [9]): $\phi(t)=K /(1-4 K t)$ for $0 \leq t<1 / 4 K$ with $K \in \mathbb{R}_{+}$. Since the function $\phi(t)$ appears in the weighted norm the inequality $\frac{\mu^{*} \phi(\tau)}{\mu^{*}-4 \phi(\tau)(t-\tau)} \leq \phi(t)$ determines the domain of existence of the unbounded solutions which satisfy the desired growth condition.

Proof of Theorem 2. We show that the operator $\mathbf{T}$ defined by the righthand side of (3) is a contraction from the space $E_{S}^{2, \phi}$ into itself with respect to the norm $\|\cdot\|_{2, \phi, \psi_{L}}$.

Let $z, \bar{z} \in E_{S}^{2, \phi}$ and $u=\mathbf{T}[z], \bar{u}=\mathbf{T}[\bar{z}]$. Owing to the definition of the mapping $\mathbf{T}$ and the operator $\mathbf{F}$,

$$
\begin{aligned}
\left|u^{i}(t, x)-\bar{u}^{i}(t, x)\right| & \leq \int_{0 \mathbb{R}^{m}}^{t} \int_{0}\left|\Gamma^{i}(t, x ; \tau, \xi)\right|\left|f^{i}(\tau, \xi, z)-f^{i}(\tau, \xi, \bar{z})\right| d \xi d \tau \\
& =\int_{0}^{t} \int_{\mathbb{R}^{m}}\left|\Gamma^{i}(t, x ; \tau, \xi)\right|\left|\mathbf{F}^{i}[z](\tau, \xi)-\mathbf{F}^{i}[\bar{z}](\tau, \xi)\right| d \xi d \tau
\end{aligned}
$$




$$
\begin{aligned}
& =\int_{0}^{t} \int_{\mathbb{R}^{m}}\left|\Gamma^{i}(t, x ; \tau, \xi)\right| \frac{\left|\mathbf{F}^{i}[z](\tau, \xi)-\mathbf{F}^{i}[\bar{z}](\tau, \xi)\right|}{\psi_{L}(\tau)[\phi(\tau)]^{m / 2} \exp \left(\phi(\tau)|\xi|^{2}\right)} \\
& \quad \times \psi_{L}(\tau)[\phi(\tau)]^{m / 2} \exp \left(\phi(\tau)|\xi|^{2}\right) d \xi d \tau .
\end{aligned}
$$

By condition $(\mathcal{L})$ and Lemma 1 one can further estimate as follows:

$$
\begin{aligned}
& \left|u^{i}(t, x)-\bar{u}^{i}(t, x)\right| \\
& \leq L\|z-\bar{z}\|_{2, \phi, \psi_{L}} \int_{0}^{t} \int_{\mathbb{R}^{m}}\left|\Gamma^{i}(t, x ; \tau, \xi)\right| \psi_{L}(\tau)[\phi(\tau)]^{m / 2} \exp \left(\phi(\tau)|\xi|^{2}\right) d \xi d \tau \\
& \leq L\|z-\bar{z}\|_{2, \phi, \psi_{L}} \int_{0}^{t} \psi_{L}(\tau)[\phi(\tau)]^{m / 2} \int_{\mathbb{R}^{m}}\left|\Gamma^{i}(t, x ; \tau, \xi)\right| \exp \left(\phi(\tau)|\xi|^{2}\right) d \xi d \tau \\
& \leq L\|z-\bar{z}\|_{2, \phi, \psi_{L}} \int_{0}^{t} \psi_{L}(\tau)[\phi(\tau)]^{m / 2} \int_{\mathbb{R}^{m}} c(t-\tau)^{-m / 2} \\
& \quad \times \exp \left(-\frac{\mu^{*}|x-\xi|^{2}}{4(t-\tau)}\right) \exp \left(\phi(\tau)|\xi|^{2}\right) d \xi d \tau .
\end{aligned}
$$

Now, from Lemma 2 we obtain

$$
\begin{aligned}
& \left|u^{i}(t, x)-\bar{u}^{i}(t, x)\right| \\
& \leq c L\|z-\bar{z}\|_{2, \phi, \psi_{L}} \int_{0}^{t} \psi_{L}(\tau)[\phi(\tau)]^{m / 2}(t-\tau)^{-m / 2}\left(\frac{4 \pi(t-\tau)}{\mu^{*}-4 \phi(\tau)(t-\tau)}\right)^{m / 2} \\
& \quad \times \exp \left(\frac{\mu^{*} \phi(\tau)|x|^{2}}{\mu^{*}-4 \phi(\tau)(t-\tau)}\right) d \tau \\
& \leq \quad c L\|z-\bar{z}\|_{2, \phi, \psi_{L}} \int_{0}^{t} \psi_{L}(\tau)\left(\frac{4 \pi \phi(\tau)}{\mu^{*}-4 \phi(\tau)(t-\tau)}\right)^{m / 2} \\
& \quad \times \exp \left(\frac{\mu^{*} \phi(\tau)|x|^{2}}{\mu^{*}-4 \phi(\tau)(t-\tau)}\right) d \tau
\end{aligned}
$$

Due to the property of the function $\phi(t)$ we have

which implies

$$
\exp \left(\frac{\mu^{*} \phi(\tau)|x|^{2}}{\mu^{*}-4 \phi(\tau)(t-\tau)}\right) \leq \exp \left(\phi(t)|x|^{2}\right)
$$

$$
\begin{aligned}
& \left|u^{i}(t, x)-\bar{u}^{i}(t, x)\right| \\
& \quad \leq c L\left(\frac{4 \pi}{\mu^{*}}\right)^{m / 2}\|z-\bar{z}\|_{2, \phi, \psi_{L}}[\phi(t)]^{m / 2} \exp \left(\phi(t)|x|^{2}\right) \int_{0}^{t} \psi_{L}(\tau) d \tau \\
& \quad \leq[\phi(t)]^{m / 2} \psi_{L}(t) \exp \left(\phi(t)|x|^{2}\right) \theta\|z-\bar{z}\|_{2, \phi, \psi_{L}} .
\end{aligned}
$$


Thus, finally,

$$
\|T[z]-T[\bar{z}]\|_{2, \phi, \psi_{L}} \leq \theta\|z-\bar{z}\|_{2, \phi, \psi_{L}} .
$$

This means that the operator $\mathbf{T}$ is a contraction with respect to the weighted norm $\|\cdot\|_{2, \phi, \psi_{L}}$. The inequality (7) also implies the boundedness of $\mathbf{T}[z]$ with respect to this norm:

$\|\mathbf{T}[z]\|_{2, \phi, \psi_{L}} \leq\|\mathbf{T}[0]\|_{2, \phi, \psi_{L}}+\|\mathbf{T}[z]-\mathbf{T}[0]\|_{2, \phi, \psi_{L}} \leq C\left(\bar{K}+M_{0} T\right)+\theta\|z\|_{2, \phi, \psi_{L}}$.

Therefore, from the Banach contraction principle it follows that there exists a unique fixed point $u=\left\{u^{i}\right\}_{i \in S}$ in $E_{S}^{2, \phi}$ of the mapping $\mathbf{T}$, which means that the problem (1), (2) has a unique $C$-solution satisfying the growth condition $\left|u^{i}(t, x)\right| \leq D \exp \left(\phi(t)|x|^{2}\right)$ in $\bar{\Omega}$. Theorem 2 is proved.

REMARK 3. In Theorem 2 we assumed that the operator generated by the right-hand sides of (1) satisfies the Lipschitz type condition with respect to the weighted norm $\|\cdot\|_{2, \phi, \psi_{L}}$. This condition is stronger than the classical one, but the example for the heat equation with functional dependence from [9] shows that the latter is not enough for the functional case when the initial data and right-hand sides are unbounded. But, in the case of non-functional dependence as well as in the case of functional dependence but in spaces of bounded continuous functions, the classical Lipschitz condition is sufficient, as was shown in [10].

If we know more about functional dependence and can effectively estimate the norm of the operator which determines that dependence, the Lipschitz condition with respect to the weighted norm can be weakened, but not to the classical one. An example is the Hale operator and the results included in [3].

As in the previous section we can allow a weak singularity at $t=0^{+}$by formulating the theorem under a Carathéodory type condition.

Assumption 4.

$\left(\mathcal{C}_{t}\right) f^{i}(t, \cdot, s) \in C\left(\mathbb{R}^{m}\right)$ and $f^{i}(\cdot, x, s) \in L^{1}[0, T]$ for all $i \in S$;

$\left(\mathcal{B}_{t}\right) \exists M_{0} \in L^{1}[0, T] \forall i \in S \forall(t, x) \in \bar{\Omega}$

$$
\left|f^{i}(t, x, 0)\right| \leq M_{0}(t) \exp \left(\phi(t)|x|^{2}\right) .
$$

Theorem 3. Let $(\mathcal{H})$ and $(\mathcal{P})$ hold. Let $\phi \in C^{+}$be a function satisfying

$$
\frac{\mu^{*} \phi(\tau)}{\mu^{*}-4 \phi(\tau)(t-\tau)} \leq \phi(t) \quad \text { for } 0 \leq \tau \leq t \leq T,
$$

where $\mu^{*}$ is the constant which appeared in Lemma 1. Let condition $(\mathcal{K})$ from Assumption 3 be satisfied and let $\psi_{L}(t):=\exp \left\{(C / \theta) \int_{0}^{t} L(\tau) d \tau\right\}$, where $0<\theta<1$ and $L \in L^{1}[0, T]$. Moreover, suppose that Assumption 4 holds and the function $f=\left\{f^{i}\right\}_{i \in S}$ satisfies the following Lipschitz condition: 


$$
\begin{aligned}
& \left(\mathcal{L}_{t}\right) \exists L \in L^{1}[0, T] \forall i \in S \forall(t, x) \in \bar{\Omega} \forall s, \widetilde{s} \in E_{S}^{2, \phi} \\
& \left|f^{i}(t, x, s)-f^{i}(t, x, \widetilde{s})\right| \leq L(t)\|s-\widetilde{s}\|_{2, \phi, \psi_{L}} \psi_{L}(t)[\phi(t)]^{m / 2} \exp \left(\phi(t)|x|^{2}\right) .
\end{aligned}
$$

Then there exists a unique solution $u \in E_{S}^{2, \phi}$ of the problem (1), (2).

We omit the proof, since it is analogous to the proof of Theorem 2.

\section{References}

[1] S. Brzychczy, On the existence of solutions of nonlinear infinite systems of parabolic differential-functional equations, Univ. Iagel. Acta Math. 40 (2002), 31-38.

[2] - Existence and uniqueness of solutions of nonlinear infinite systems of parabolic differential-functional equations, Ann. Polon. Math. 77 (2001), 1-9.

[3] A. Bychowska, Existence of unbounded solutions to parabolic equations with functional dependence, Math. Nachr. 263-264 (2004), 53-66.

[4] A. Bychowska and H. Leszczyński, Parabolic equations with functional dependence, Z. Anal. Anwendungen 20 (2001), 115-130.

[5] J. Dugundji and A. Granas, Fixed Point Theory, Vol. 1, Monograf. Mat. 61, PWN, Warszawa, 1982.

[6] S. D. Eidel'man, Parabolic Systems, North-Holland, 1969.

[7] A. Friedman, Partial Differential Equations of Parabolic Type, Prentice-Hall, Englewood Cliffs, NJ, 1964.

[8] O. A. Ladyzhenskaya, V. A. Solonnikow and N. N. Ural'tseva, Linear and Quasilinear Equations of Parabolic Type, Nauka, Moscow, 1967 (in Russian); English transl.: Math. Monogr. 23, Amer. Math. Soc., Providence, RI, 1968.

[9] H. Leszczyński, A new existence result for a non-linear heat equation with functional dependence, Ann. Polon. Math. 37 (1997), 155-181.

[10] A. Pudełko, Existence and uniqueness of solutions [of the] Cauchy problem for nonlinear infinite systems of parabolic differential-functional equations, Univ. Iagel. Acta Math. 40 (2002), 49-56.

[11] A. N. Tikhonov, Théorèmes d'unicité pour l'équation de la chaleur, Mat. Sb. 42 (1935), 199-216.

Faculty of Applied Mathematics

AGH University of Science and Technology

Al. Mickiewicza 30

30-059 Kraków, Poland

E-mail: fronczyk@wms.mat.agh.edu.pl 